\title{
Specificity of brachyury in the distinction of chordoma from clear cell renal cell carcinoma and germ cell tumors: a study of $\mathbf{3 0 5}$ cases
}

\author{
Ankur R Sangoi ${ }^{1,2}$, Jason Karamchandani ${ }^{1}$, Brooke Lane ${ }^{1}$, John P Higgins ${ }^{1}$, \\ Robert V Rouse ${ }^{1,3}$, James D Brooks ${ }^{4}$ and Jesse K McKenney ${ }^{1,4}$ \\ ${ }^{1}$ Department of Pathology, Stanford University, Stanford, CA, USA; ${ }^{2}$ Department of Pathology, El Camino \\ Hospital, Mountain View, CA, USA; ${ }^{3}$ Department of Pathology, Veterans Affairs Palo Alto Medical Center, \\ Palo Alto, CA, USA and ${ }^{4}$ Department of Urology, Stanford University, Stanford, CA, USA
}

\begin{abstract}
Brachyury is recognized as a specific marker for notochord-derived tissues and neoplasms, and has become a defining immunohistochemical feature of chordoma. The main differential diagnostic consideration for chordoma is chondrosarcoma, which is known to lack brachyury expression. However, within the spectrum of genitourinary neoplasia, metastatic germ cell tumors and clear cell renal cell carcinoma may also be close morphological mimics of chordoma, particularly given the increasing prevalence of small tissue samples from image-guided biopsies. Although immunoreactivity for brachyury has been reported in a few germ cell tumors, a thorough characterization of staining by specific subtype has not been performed in a large series. Additionally, brachyury expression in clear cell renal cell carcinoma has not been well studied. In this study, immunohistochemical expression with the brachyury antibody was evaluated in 111 germ cell tumors, 30 non-neoplastic and neoplastic (non-germ cell) testicular tissues, and 184 metastatic clear cell renal cell carcinomas using tissue microarray technology. In addition, immunoreactivity for PAX-8 and SALL-4 was evaluated in 12 chordomas on whole section. No nuclear brachyury expression was identified in any of the 101 germ cell tumors within the tissue microarray (including choriocarcinoma (1), embryonal carcinoma (20), intratubular germ cell neoplasia unclassified (2), seminoma (64), spermatocytic seminoma (1), teratoma (5) and yolk sac tumor (8)), in any of the $\mathbf{3 0}$ non-neoplastic and neoplastic (non-germ cell) testicular tissues, or in any of the 10 whole-section seminomas. All 184 metastatic clear cell renal cell carcinomas were also non-reactive for brachyury. All 12 chordomas showed strong nuclear immunoreactivity for brachyury, but no expression of SALL-4. In all, 1 of 12 chordoma cases showed patchy, $1+$ nuclear immunoreactivity for PAX-8. This study confirms the specificity of brachyury for chordoma in the differential diagnostic distinction from the potential genitourinary mimics, germ cell tumors and metastatic clear cell renal cell carcinoma.

Modern Pathology (2011) 24, 425-429; doi:10.1038/modpathol.2010.196; published online 19 November 2010
\end{abstract}

Keywords: brachyury; chordoma; germ cell tumor; metastatic; renal cell carcinoma

Brachyury is recognized as a key transcription factor expressed in notochord-derived tissues and neoplasms. Although expression of the novel antigen brachyury has come to be considered a defining diagnostic feature of chordoma, ${ }^{1-6}$ few studies have thoroughly addressed specificity of the antibody. Chondrosarcoma, the most recognized differential diagnostic consideration for chordoma, has

Correspondence: Dr AR Sangoi, MD, Department of Pathology, El Camino Hospital, GC-33, Mountain View, CA, 94040, USA. E-mail: asangoi2@yahoo.com

Received 15 July 2010; revised 30 August 2010; accepted 30 August 2010; published online 19 November 2010 been thoroughly evaluated in previous studies and does not express brachyury. ${ }^{2,5-9}$ However, within the spectrum of genitourinary neoplasia, metastatic germ cell tumors and clear cell renal cell carcinomas may also be close morphological mimics of chordoma, particularly on a small biopsy sample. Immunoreactivity with the brachyury antibody has been reported in a few germ cell tumors, either in cases without reported subtype ${ }^{5}$ or in seminomas; ${ }^{3}$ however, a full characterization of staining by tumor subtype has not been evaluated in a large series. Additionally, brachyury expression in clear cell renal cell carcinoma has not been thoroughly studied. 


\section{Materials and methods}

In all, 111 germ cell tumors, 184 metastatic clear cell renal cell carcinomas and 12 chordomas were retrieved from the pathology archives of Stanford University Medical Center and/or Veterans Affairs Palo Alto Medical Center. Standard whole sections from each were reviewed and diagnoses for all cases were confirmed on routine hematoxylin and eosinstained sections. A tissue microarray consisting of 101 germ cell tumors (including choriocarcinoma (1), embryonal carcinoma (20), intratubular germ cell neoplasia unclassified (2), seminoma (64), spermatocytic seminoma (1), teratoma (5) and yolk sac tumor (8)) using $1.2 \mathrm{~mm}$ diameter tissue cores was prepared (Stanford tissue microarray 136) and evaluated as described in detailed elsewhere. ${ }^{10}$ Similarly, a tissue microarray of 184 metastatic clear cell renal cell carcinomas from 26 unique sites using $0.7 \mathrm{~mm}$ diameter tissue cores was prepared (in duplicate) and evaluated as above (Stanford tissue microarray 238). Only metastatic clear cell renal cell carcinoma cases with a documented prior primary renal cell carcinoma history (by chart review or in-house pathology database review) were included in the study. Additionally, although some cases had synchronous metastasis in other organs/sites (eg, lung plus multiple lymph node metastasis), only one representative block per patient was selected to eliminate data duplication. Immunohistochemical staining using anti-brachyury antibody (rabbit polyclonal (1:50); Santa Cruz Biotechnology, Santa Cruz, CA, USA; Ventana Benchmark XT (Tuscon, AZ, USA) autostainer with EDTA retrieval) was performed using standard avidin-biotin technique on 4-mm thick formalin-fixed, paraffin-embedded tissue microarray sections mounted on charged slides baked at $60^{\circ} \mathrm{C}$ for $1 \mathrm{~h}$. To address the potential staining heterogeneity bias of a tissue microarray study, 10 whole sections of seminomas with associated intratubular germ cell neoplasia unclassified were also stained for brachyury, with the diagnoses confirmed by immunostaining for OCT3/4 (anti-OCT3/4; goat polyclonal (1:400); Santa Cruz Biotechnology; Leica Bond Max (Bannockburn, IL, USA) autostainer with citrate retrieval). Whole sections of 12 classic chordomas were used as positive control tissue, and only nuclear reactivity was scored as positive. Expression of PAX-8 (anti-PAX8, rabbit polyclonal antibody (1:20); Proteintech, Chicago, IL, USA; Dako (Carpinteria, CA, USA) autostainer with citrate retrieval) and SALL-4 (anti-SALL4, clone 6E3 (1:200); Abnova, Walnut, CA, USA; Ventana Benchmark XT autostainer with EDTA retrieval) was also evaluated in the 12 control chordomas cases. In all, 30 non-neoplastic and non-germ cell neoplastic testicular tissues present on the germ cell tumor tissue microarray were used as negative controls. In all, 10 non-germ cell tumor malignancies and 5 non-neoplastic tissues were used as negative controls for the metastatic clear cell renal cell carcinoma tissue microarray.

\section{Results}

No nuclear brachyury expression was identified in any of the 111 germ cell tumors, including 101 tissue microarray cases and 10 whole sections of seminomas (Figure 1) (Table 1). In the 10 whole sections of seminoma, brachyury expression was also not identified in surrounding non-neoplastic testis. Similarly, brachyury expression was not identified in any of the 30 non-germ cell tumor testicular tissues present on the germ cell tumor microarray (including adenomatoid tumor (1), atrophy (7), cryptorchidism (1), fibrous pseudotumor (1), hypospermatogenesis (1), inflamed testis (2), Leydig cell hyperplasia (1), Leydig cell tumor (1), liposarcoma (3), normal epididymis (2), normal testis (9) and serous papillary carcinoma (1)). All 184 metastatic clear cell renal cell carcinomas from 26 unique sites were non-reactive for brachyury (Figure 1) (Table 2). In all, 10 non-germ cell tumor malignancies and 5 non-neoplastic tissues present on the metastatic clear cell renal cell carcinoma array were also negative for brachyury (including colorectal adenocarcinoma (1), hepatocellular carcinoma (1), lung adenocarcinoma (1), mammary ductal carcinoma (1), melanoma (1), normal lymph node (1), normal placenta (4), ovarian clear cell carcinoma (1), prostatic adenocarcinoma (2), urinary bladder carcinoma (1) and uterine cervical adenocarcinoma (1)). All 12 chordomas showed nuclear reactivity for brachyury, but no expression with anti-SALL-4. In all, 1 of 12 chordomas showed focal $1+$ nuclear PAX-8 immunoreactivity.

\section{Discussion}

mRNA expression of brachyury, a T-box mesodermal transcription factor critical in the development of notochord-derived tissues and neoplasms, has been demonstrated in various human carcinomas; ${ }^{11}$ however, few studies have comprehensively explored immunohistochemical expression of brachyury in other tissues to address the diagnostic specificity for chordoma in non-notochord derived tumors with clear cytoplasm that may be close morphological mimics. ${ }^{5-7}$ Although the morphological overlap between chordoma and clear cell renal cell carcinoma is well known, we have also encountered cases in which the distinction from central nervous system germ cell tumors such as seminoma and yolk sac tumor was considered, especially in the evaluation of poorly differentiated (anaplastic) chordomas. Within the spectrum of genitourinary neoplasia, rare brachyury immunoreactivity has been reported in testicular germ cell tumors (2/14 cases; most unspecified as to subtype), including focally within non-neoplastic testis., ${ }^{5,7}$ A study presented in abstract form has also reported focal brachyury staining in four out of six seminomas, ${ }^{3}$ whereas a very recent study reported no staining in six additional testicular germ cell tumors (four 


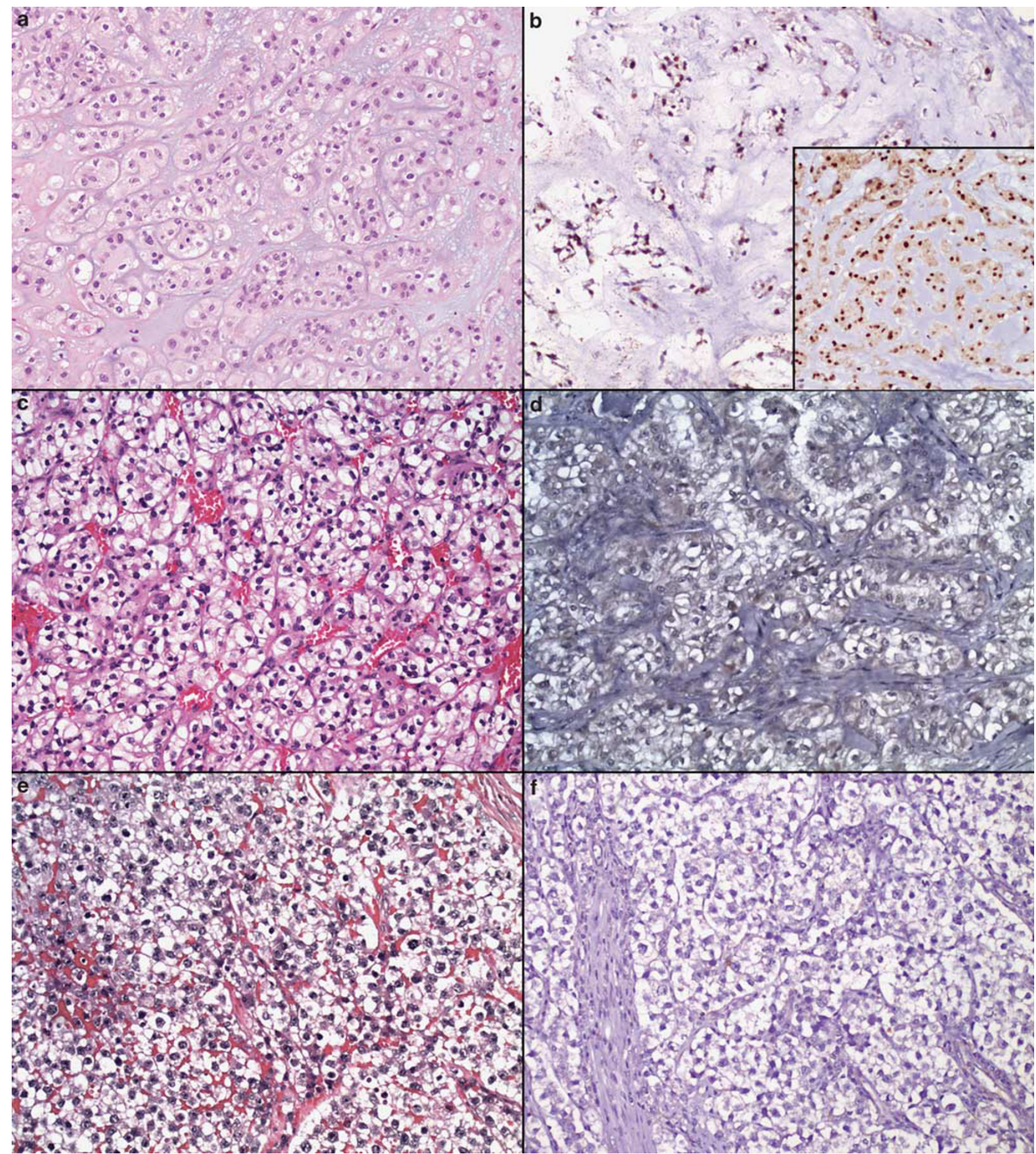

Figure 1 (a) Hematoxylin and eosin (H\&E), nested and cord-like growth typical of chordoma with intracytoplasmic vacuolization; (b) strong nuclear immunoreactivity for brachyury was seen in all 12 chordoma cases (highlighted in high power inset); (c) H\&E, clear cell renal cell carcinoma showing the characteristic nested pattern with surrounding vascular septa; (d) no immunoreactivity for brachyury was seen in renal cell carcinoma $(n=184)$; (e) H\&E, classic seminoma with clear cytoplasm; (f) no immunoreactivity for brachyury was seen in any of the germ cell tumors $(n=111)$.

seminomas and two mixed germ cell tumors). ${ }^{7}$ The current study was undertaken because of the potential morphological overlap of chordoma with these genitourinary tumors, the emerging recognition of true primary peripheral chordomas in unusual sites, ${ }^{5,8,12,13}$ and the lack of a large compre- hensive series of cases examined for brachyury expression.

In this study, brachyury expression was not identified in any of the 111 germ cell tumors or 30 non-germ cell tumor testicular tissues investigated, including a lack of staining in the surrounding 
Table 1 Immunostaining results with anti-brachyury antibody in germ cell tumors

\begin{tabular}{lc}
\hline Germ cell tumor & Positive cases \\
\hline Choriocarcinoma & $0 / 1$ \\
Embryonal carcinoma & $0 / 19$ \\
Embryonal carcinoma (pure) $_{\text {ITGCNU }^{\mathrm{a}}}$ & $0 / 1$ \\
Seminoma $_{\text {Seminoma (pure) }}$ & $0 / 2$ \\
Seminoma+ITGCNU $^{\mathrm{b}}$ & $0 / 52$ \\
Spermatocytic seminoma & $0 / 12$ \\
Teratoma (immature) & $0 / 10$ \\
Teratoma (mature) & $0 / 1$ \\
Yolk sac tumor & $0 / 2$ \\
Total & $0 / 3$ \\
& $0 / 8$ \\
\hline
\end{tabular}

${ }^{\mathrm{a}}$ Intratubular germ cell neoplasia unclassified.

${ }^{\mathrm{b}}$ Whole-section analysis.

non-neoplastic testis of 10 whole-section seminomas. Although this finding contrasts with other reports of brachyury immunoreactivity in germ cell tumors (both seminoma ${ }^{3}$ and unspecified subtypes ${ }^{5}$ ) and non-neoplastic testis (spermatogonia), ${ }^{5,7}$ it should be noted that both studies describe and/or depict immunoreactivity that is very focal in nature. Given the reports of high brachyury mRNA expression in testicular carcinoma (type unspecified) with low level expression in normal testis, ${ }^{11}$ theoretically, it would not be totally unexpected to find immunoreactivity for brachyury in paraffin sections. The different results in these studies may potentially be secondary to differences in staining methodology; however, our chordoma controls showed strong and diffuse nuclear reactivity with brachyury and we utilized both the same antibody and the same dilution as reported previously. In order to address potential sampling bias in our study due to the evaluation of small core tissue samples, we also evaluated brachyury staining in whole-section seminomas. Despite the fact that seminoma is the subtype most frequently reported to show brachyury staining, we again found no immunoreactivity in these additional cases.

Similarly, immunohistochemical expression of brachyury was not identified in any of the 184 metastatic clear cell renal cell carcinomas. Brachyury immunoreactivity in renal cell carcinoma has, to the authors' knowledge, previously been studied in only one series with six cases that were all negative. ${ }^{6}$ Particularly, given the rising incidence of metastatic renal cell carcinoma, ${ }^{14,15}$ the increasing use of targeted therapies, and the propensity for metastasis to virtually any body site, ${ }^{16,17}$ distinction of metastatic clear cell renal cell carcinoma from chordoma is critical. Moreover, the high incidence of bony metastasis in this study (Table 2), an incidence supported by a previous study, ${ }^{16}$ makes metastatic clear cell renal cell carcinoma a particularly plausible morphological mimic of chordoma (both axial and extra-axial), particularly on a small biopsy sample.
Table 2 Immunostaining results with anti-brachyury antibody in metastatic CC-RCC ${ }^{\text {a }}$

\begin{tabular}{lc}
\hline Metastatic CC-RCC site & Positive cases \\
\hline Bone & $0 / 39$ \\
Lung & $0 / 38$ \\
Lymph node & $0 / 30$ \\
Soft tissue & $0 / 17$ \\
Brain & $0 / 10$ \\
Vessel & $0 / 7$ \\
Skin & $0 / 6$ \\
Adrenal & $0 / 5$ \\
Parotid & $0 / 5$ \\
Pleura & $0 / 4$ \\
Nose/nasal & $0 / 3$ \\
Bronchus & $0 / 2$ \\
Chest wall & $0 / 2$ \\
Diaphragm & $0 / 2$ \\
Pancreas & $0 / 2$ \\
Breast & $0 / 1$ \\
Heart & $0 / 1$ \\
Small bowel & $0 / 1$ \\
Liver & $0 / 1$ \\
Omentum & $0 / 1$ \\
Pituitary & $0 / 1$ \\
Retroperitoneum & $0 / 1$ \\
Spleen & $0 / 1$ \\
Testicle & $0 / 1$ \\
Thyroid & $0 / 1$ \\
Tonsil & $0 / 1$ \\
Total & $0 / 184$ \\
\hline
\end{tabular}

${ }^{\mathrm{a}}$ Clear cell renal cell carcinoma.

The main goal of this study was to confirm the specificity of brachyury for chordoma in the differential diagnostic distinction from these metastatic genitourinary mimics. Other immunohistochemical stains would obviously be useful adjuncts in this differential diagnostic setting. The widely available renal cell carcinoma marker PAX-8 $8^{18,19}$ and the novel germ cell marker SALL- $4^{20-22}$ would be useful in an immunohistochemical panel when renal cell carcinoma or a germ cell tumor are considered, respectively. Although no chordomas expressed SALL-4 in this study, 1 of 12 cases had patchy weak nuclear immunoreactivity for PAX-8. This finding underscores the importance of using a panel of antibodies in this differential diagnostic setting.

In conclusion, unlike previous results, this study confirms the specificity of the brachyury antibody for chordoma in the differential diagnostic distinction from the genitourinary mimics clear cell renal cell carcinoma and germ cell tumors.

\section{Acknowledgement}

We acknowledge Shirley Kwok and Kelli Montgomery for excellence in tissue microarray construction.

\section{Disclosure/conflict of interest}

The authors declare no conflict of interest. 


\section{References}

1 Henderson SR, Guiliano D, Presneau N, et al. A molecular map of mesenchymal tumors. Genome Biol 2005;6:R76.

2 Oakley GJ, Fuhrer K, Seethala RR. Brachyury, SOX-9, and podoplanin, new markers in the skull base chordoma vs chondrosarcoma differential: a tissue microarray-based comparative analysis. Mod Pathol 2008;21:1461-1469.

3 Rekhi B, Thorat K, Jambhekar NA, et al. Utility of brachyury as a 'new-age' marker in the diagnosis of chordomas. Mod Pathol 2009;22:21A (Abstract 82).

4 Romeo S, Hogendoorn PC. Brachyury and chordoma: the chondroid-chordoid dilemma resolved? J Pathol 2006;209:143-146.

5 Tirabosco R, Mangham DC, Rosenberg AE, et al. Brachyury expression in extra-axial skeletal and soft tissue chordomas: a marker that distinguishes chordoma from mixed tumor/myoepithelioma/parachordoma in soft tissue. Am J Surg Pathol 2008;32:572-580.

6 Vujovic S, Henderson S, Presneau N, et al. Brachyury, a crucial regulator of notochordal development, is a novel biomarker for chordomas. J Pathol 2006;209:157-165.

7 Jambhekar NA, Rekhi B, Thorat K, et al. Revisiting chordoma with brachyury, a 'new age' marker: analysis of a validation study on 51 cases. Arch Pathol Lab Med 2010;134:1181-1187.

8 O’Donnell P, Tirabosco R, Vujovic S, et al. Diagnosing an extra-axial chordoma of the proximal tibia with the help of brachyury, a molecule required for notochordal differentiation. Skeletal Radiol 2007;36:59-65.

9 Sangoi AR, Dulai MS, Beck AH, et al. Distinguishing chordoid meningiomas from their histologic mimics: an immunohistochemical evaluation. Am J Surg Pathol 2009;33:669-681.

10 Kononen J, Bubendorf L, Kallioniemi A, et al. Tissue microarrays for high-throughput molecular profiling of tumor specimens. Nat Med 1998;4:844-847.

11 Palena C, Polev DE, Tsang KY, et al. The human T-box mesodermal transcription factor Brachyury is a candidate target for T-cell-mediated cancer immunotherapy. Clin Cancer Res 2007;13:2471-2478.
12 Nielsen GP, Mangham DC, Grimer RJ, et al. Chordoma periphericum: a case report. Am J Surg Pathol 2001;25:263-267.

13 Suster S, Moran CA. Chordomas of the mediastinum: clinicopathologic, immunohistochemical, and ultrastructural study of six cases presenting as posterior mediastinal masses. Hum Pathol 1995;26: 1354-1362.

14 Decastro GJ, McKiernan JM. Epidemiology, clinical staging, and presentation of renal cell carcinoma. Urol Clin North Am 2008;35:581-592; vi.

15 Gupta K, Miller JD, Li JZ, et al. Epidemiologic and socioeconomic burden of metastatic renal cell carcinoma (mRCC): a literature review. Cancer Treat Rev 2008;34:193-205.

16 Hoffmann NE, Gillett MD, Cheville JC, et al. Differences in organ system of distant metastasis by renal cell carcinoma subtype. J Urol 2008;179:474-477.

17 Wahner-Roedler DL, Sebo TJ. Renal cell carcinoma: diagnosis based on metastatic manifestations. Mayo Clin Proc 1997;72:935-941.

18 McKenney JK, Fujiwara M, Higgins JP, et al. Comparison of putative renal cell carcinoma markers in primary adrenal cortical lesions and metastatic renal cell carcinoma: a tissue microarray study of 246 cases. Mod Pathol 2009;22:182A (Abstract 822).

19 Tong GX, Yu WM, Beaubier NT, et al. Expression of PAX8 in normal and neoplastic renal tissues: an immunohistochemical study. Mod Pathol 2009;22: 1218-1227.

20 Cao D, Humphrey PA, Allan RW. SALL4 is a novel sensitive and specific marker for metastatic germ cell tumors, with particular utility in detection of metastatic yolk sac tumors. Cancer 2009;115:2640-2651.

21 Mei K, Liu A, Allan RW, et al. Diagnostic utility of SALL4 in primary germ cell tumors of the central nervous system: a study of 77 cases. Mod Pathol 2009;22:1628-1636.

22 Wang F, Liu A, Peng Y, et al. Diagnostic utility of SALL4 in extragonadal yolk sac tumors: an immunohistochemical study of 59 cases with comparison to placental-like alkaline phosphatase, alpha-fetoprotein, and glypican-3. Am J Surg Pathol 2009;33:1529-1539. 